\title{
An Optimized WSN Design for Latency-Critical Smart Grid Applications
}

\author{
Mounib Khanafer, ${ }^{1}$ Irfan Al-Anbagi, ${ }^{2}$ and Hussein T. Mouftah ${ }^{3}$ \\ ${ }^{1}$ College of Arts and Sciences, American University of Kuwait, Salmiya, Kuwait \\ ${ }^{2}$ Faculty of Engineering and Applied Science, University of Regina, Regina, SK, Canada \\ ${ }^{3}$ School of Electrical Engineering and Computer Science, University of Ottawa, Ottawa, ON, Canada \\ Correspondence should be addressed to Irfan Al-Anbagi; alanbagi@uregina.ca
}

Received 30 January 2017; Revised 10 May 2017; Accepted 25 May 2017; Published 2 July 2017

Academic Editor: Raymond Swartz

Copyright (C) 2017 Mounib Khanafer et al. This is an open access article distributed under the Creative Commons Attribution License, which permits unrestricted use, distribution, and reproduction in any medium, provided the original work is properly cited.

\begin{abstract}
The growing popularity of the Internet of Things (IoT) systems such as the smart grid, Body Area Networks (BANs), and the Intelligent Transportation System (ITS) is driving Wireless Sensor Network (WSN) systems to the limit in terms of abilities and performance. WSNs were initially designed for low power, low data rate, and latency-tolerant applications. However, this paradigm is changing because of the nature of the new applications. Therefore, instead of only focusing on power-efficient WSN design, researchers and industries are now developing Quality of Service (QoS) protocols for WSNs. In addition to that, latency- and reliability-critical protocol designs are also becoming significantly important in WSNs. In this paper, we present an overview of some important smart grid latency-critical applications and highlight WSNs implementation challenges for these smart grid applications. Furthermore, we develop and evaluate two novel optimization models that solve for the optimum values of the end-to-end latency and power consumption in a clustered WSN given lower bounds on reliability and other network parameters.
\end{abstract}

\section{Introduction}

Internet of Things (IoT) systems significantly rely on sensors and sensor networks for their successful operation. Examples of IoT applications include, but are not limited to, the smart grid, Body Area Networks (BANs), Intelligent Transportation Systems (ITSs), battlefield surveillance, object movement detection, disaster discovery, and security systems. So far, there has not been a published standard that governs and regulates the IoT framework. The IEEE Standards Association (IEEE-SA) is developing a number of standards and projects that are associated with creating the environment needed for a dynamic IoT framework. On the other hand, the IEEE 802.15.4 (or 802.15.4 hereafter) standard [1] outlines the features and the operating conditions of the physical layer (PHY) and the Medium Access Control (MAC) sublayer of the Low-Rate Wireless Personal Area Networks (LR-WPAN). It is the main standard used in Wireless Sensor Networks (WSNs). WSNs are popular because of the wide range of IoT applications they can be used in. In effect, WSNs are being implemented in almost all IoT applications due to their effectiveness in transmitting many monitoring many events. Therefore, we can rely on the 802.15.4 standard for developing an IoT framework using WSNs. WSNs are built from small, sensor nodes that can monitor and collect data (temperature, vibration, motion, etc.), process that data, and communicate it wirelessly to a sink node or a Base Station (BS).

WSNs are plentiful and go more than a decade back. In the literature, there are numerous studies that examined different areas of WSN technologies and their applications [2-5]. Sensor nodes are battery-powered and usually deployed in environments that have least human supervision (like forests, battlefields, etc.). As such, replacing or recharging the sensor nodes batteries is not practical. In order to avoid any disruption in the services of the WSN due to battery drainage, two approaches are followed. From one side, sensor nodes are deployed in abundance (WSNs can be constituted by thousands of sensor nodes) in order to guarantee redundancy in the data reported by the sensor nodes. That is, if certain sensor nodes deplete their batteries, other nodes are already 
covering for them and serious disruption in services is mitigated. On the other side, any communication protocol or algorithm to be ported on the sensor node platform should not pose any processing burden. The early phases of design, development, and deployment of WSNs focused on conserving the power resources of the sensor nodes (see $[3,5]$ for details). There are a number of studies that consider optimizing the power consumption of WSNs or optimizing certain parameters of the 802.15.4 standard, under power consumption constraints [6-9]. With the development of high efficiency energy harvesting mechanisms, sensor nodes can harvest enough power to continue with their operation. As an example, [10] presents a comprehensive review of energy harvesting systems. Therefore, the design approaches of WSNs can relax the constraints on power consumption, or assume that an energy harvesting is implemented. Furthermore, because many IoT applications [5] have certain requirements on reliability and latency, WSNs are required to focus on not only minimizing the power consumption, but also minimizing the latency and maximizing the reliability levels. These requirements change how we formulate optimization problems in the context of 802.15.4-based WSNs. The nature of the application and the available power sources make the constraint on power consumption less stringent compared to latency and reliability. Minimizing the delay and maximizing the reliability enabled new applications to benefit from the unique features of WSNs. This is more important in latency- and reliability-critical environments that need strict Quality of Service (QoS) requirements. In this paper, we choose the smart grid environment as an example of a latency-critical IoT application to design our WSN.

In this paper, we present an overview of some important smart grid latency-critical applications and highlight WSNs implementation challenges for these applications. Furthermore, we present and evaluate two novel optimization models that find the optimum values of the end-to-end latency and power consumption in a clustered WSN given certain bounds on reliability and other network parameters. We formulate two optimization problems that focus on the two parameters as follows:

(1) Minimizing the end-to-end latency with constraints on the reliability and cluster size (while relaxing the power consumption constraint).

(2) Minimizing the power consumption with constraints on the reliability and cluster size.

The first problem is important in latency-critical smart grid applications. According to [11], Utility operations require that timely actions be taken in controlling the grid elements when faults or other anomalous behaviors are observed based on grid measurements. This means that different smart grid applications can tolerate different end-to-end latencies. This requirement significantly impacts the network performance. As an example, teleprotection applications can only allow a few milliseconds, between the time a transmission line fault is detected and the tripping of a circuit breaker [11]. On the other hand, other applications, like recloser systems, Phasor Measurement Units (PMUs), and synchrophasors, can accept latencies between $20 \mathrm{~ms}$ and $100 \mathrm{~ms}$. Based on that, to solve the first optimization problem (the latency requirement), we develop an optimization problem to achieve the minimum end-to-end latency while keeping certain levels of reliability, power consumption, number of nodes, and other MAC parameters defined in the 802.15.4 standard [1].

The interest in the second problem stems from the fact that given the self-organizing nature of the WSN, and in the absence of energy harvesting technologies, it is interesting to see the bounds on power consumption given a certain minimum reliability to achieve.

The rest of this paper is organized as follows. In Section 2, we highlight related works that tackled the problem of optimizing the performance of WSNs. In Section 3, we present an overview of smart grid latency-critical applications and some implementation challenges. In Section 4, we provide full details on the formulation of the aforementioned optimization problems. In Section 5, we present the results generated from solving the optimization problems along with discussions and comments. Finally, Section 6 concludes this paper.

\section{Related Work}

In the literature, there have been a number of models that attempted to optimize the end-to-end latency, energy efficiency, or the reliability in WSNs for certain latencyand reliability-critical applications [12-17]. In the past, the majority of the work focused on minimizing the energy consumption in WSNs while setting either the latency or the reliability as a constraint. The introduction of WSNs for certain applications such as device-to-device communication, smart grid monitoring, and other industrial monitoring applications calls for high reliability and strict real-time requirements.

Mohrehkesh and Weigle [12] have developed a model that finds the optimum combination of WSNs parameters to minimize energy consumption while satisfying the latency and reliability requirements. Their method uses packet size and code weight for optimization and considers the energy consumption for transmitter and receiver. Ammari [13] has developed a data forwarding protocol that minimizes the energy consumption and the latency via slicing the communication range of the sensors into coordinated circular bands. The author presented a weighted scale-uniform-unit sum approach used by the source sensors to solve his multiobjective optimization problem.

Suto et al. [14] have proposed an energy-efficient and latency-aware wireless computing system. Their proposed system controls the sleep schedule and the number of links to minimize the power consumption while satisfying an acceptable latency constraint. He et al. [15] have proposed a WSN planning method using three-dimensional ray tracing method to model the radio link and sensing signal. They have developed a multiobjective optimization algorithm to meet basic characteristics of WSNs.

Zonouz et al. [16] have developed a model to find the optimal locations for a specific number of energy harvesting sensor nodes in the hybrid WSN to provide the optimum 
TABLE 1: Delay and QoS requirements of smart grid applications [11].

\begin{tabular}{lcc}
\hline Application & Delay requirements $(\mathrm{ms})$ & Priority (0: max to 100: min) \\
\hline Teleprotection (for $60 \mathrm{~Hz}, 50 \mathrm{~Hz})$ & 8,10 & 2 \\
$\begin{array}{l}\text { Breaker reclosers } \\
\text { Phasor measurement unit data + status } \\
\text { (class A) for other than protection }\end{array}$ & 16 & 15 \\
$\begin{array}{l}\text { Distributed automation periodic } \\
\text { measurement + status, events, control }\end{array}$ & 100 & 26 \\
$\begin{array}{l}\text { Dynamic Line Rating } \\
\text { AMI Priority }\end{array}$ & 200 & 28 \\
Transformer protection and control & 300 & 70 \\
applications & 500 & 80 \\
Fault recorders & 1000 & 90 \\
Demand response applications & 1000 & 90 \\
Best effort traffic & 2000 & 100 \\
\hline
\end{tabular}

combination of average path reliability, path cost, and average energy consumption. Dong et al. [17] have presented a data gathering protocol based on the requirements of a sensing application. They have done this through trade-offs between the energy consumption and source-to-sink transport latency under reliability constraint. Their protocol achieves energy and latency efficiency during the data gathering process both in intracluster and intercluster.

In our proposed model, we consider two scenarios. In the first scenario, an appropriate energy harvesting technique is assumed to be used and thus power consumption is not specified as a constraint in the optimization problem. The objective is to minimize the end-to-end latency given a minimum reliability and other MAC parameter constraints. In the second scenario, we study the minimum possible power consumption given a minimum reliability.

\section{Overview of Smart Grid Latency-Critical Applications}

3.1. Smart Grid Latency-Critical Applications. The use of WSNs in a smart grid environment is governed by the latency and the reliability requirements of different applications. Most of these applications require very high reliability, but they significantly vary in terms of their latency and priority requirements. For example, teleprotection systems, emergency power restoration, and substation monitoring require different delay requirements compared to Advanced Metering Infrastructure (AMI) and protection operations. On the other hand, the priority or QoS requirements also vary depending on the criticality of the monitored power grid component. In previous work, we focused on guaranteeing the QoS in WSNs [18]. In this paper, we focus on optimizing the latency for given network and traffic conditions.

Table 1 shows typical delay and QoS requirements of selected smart grid applications [11]. The delay requirements shown in the paper represent end-to-end delays (one way). These delay requirements are specified in different standards that govern the grid operations. A closer look at Table 1 shows that ZigBee-based WSNs can not satisfy certain smart applications (e.g., Teleprotection (for $60 \mathrm{~Hz}, 50 \mathrm{~Hz}$ )). This is because ZigBee or even ZigBee Pro.-based sensor networks are limited in bandwidth. However, with careful sensor design and by using optimization algorithms WSNs can support many of the applications listed in Table 1.

There are several factors that impact the delay of data transmission in a smart grid environment, such as the size of the network, the packet arrival rate, and the packet size. Denser WSNs suffer from excessive collisions and higher delays. Transmission at a high packet rate will also cause collisions in the network. Packet sizes also impact the delay behavior in the network because the packets need to be stored in the buffers of intermediate nodes. Transmitting data in smaller packet sizes results in lower delays. However, this increases transmission rates and that may cause higher delays due to collisions. The trade-off between packet size and transmission rate has been discussed in the literature. In addition to the above factors the implementation of network security algorithms may cause additional delays. In many cases smart grid applications dictate the network size, packet transmission rate, and the necessity to implement a security mechanism. Hence, we are left with a few options to minimize the delay using the above factors. Therefore, it is important to focus on the internal architecture of WSN protocols to achieve the delay requirements without impacting the above factors. In any case, smart grid applications are not concerned with how the protocols work as long as the requirements are met. Based on that, the focus turn to optimizing (minimizing) the delay given certain constraints and tuning parameters that are within the scope of the 802.15.4 standard.

3.2. WSNs Smart Grid Implementation Challenges. There are a number of challenges that need to be overcome to implement WSNs in latency-critical smart grid applications. Some of these challenges can be seen in some other applications. As mentioned above, smart grid environments pose unique challenges due to the criticality of the application. We present some of the challenges related to implementing delay minimization approaches in smart grid applications. These challenges can be summarized as follows: 
(1) The limitations on sensor nodes' resources including the available energy, computational power, and bandwidth are the main challenging issues for implementing WSNs for latency-critical smart grid applications. A balance between energy efficiency and delay minimization in smart grid applications is an interesting problem where WSN protocols are required to identify the architecture for minimizing the end-to-end delay.

(2) The use of different WSNs data dissemination techniques may impact the end-to-end delay in a delayconstrained smart grid application. The use of a realtime data dissemination is another challenge.

(3) The impact of high traffic intensities generated by sensor devices on the end-to-end delay is also a challenge that needs to be overcome for latencycritical smart grid applications.

(4) Maintaining consistent performance throughout the network life while considering optimizing the energy consumption and the end-to-end delay.

(5) Achieving a feasible solution for a minimum endto-end delay problem with multiple constraints such as energy, memory, processing capability, adaptively balancing between the amount of transmitted traffic, application requirement, and the available resources.

(6) WSN scalability and the end-to-end delay limits are major challenges in achieving the delay requirement for latency-critical smart grid application. These challenges are more significant when deploying the WSN in wide area scenarios.

(7) Another challenge is that the implementation of a reliable security algorithm may impact the end-toend delay performance because of the additional computational cycles imposed on a resource constrained sensor node.

\section{End-to-End Delay Optimization Problem Formulation}

The Markov-based mathematical model of the IEEE 802.11 MAC protocol that was proposed by Bianchi [19] motivated a massive number of proposals to devise similar models for the 802.15.4 MAC protocol. This is because both standards have major commonalities. An interesting work was introduced by Park et al. [20]. In the rest of this paper, we discuss how we benefit from the formulation that was developed in that work in developing two optimization problems. In particular, we depend on the formulae of the end-to-end latency, reliability, and the total power consumption that were derived in [20] (the details of the derivations are omitted and the interested reader can find all the details in [20]). All parameters used in the following discussion are listed in Table 2. The time $(T)$ needed for communication between a cluster member and its Cluster Head $(\mathrm{CH})$ is composed of three time durations, namely, the backoff time $\left(T_{\mathrm{BO}}\right)$, the time needed to successfully transmit a packet $(L)$, and the time lost due to having $j$ collisions $(j L)$. The following formulae reflect all these time durations:

$$
T=(1+j) L+T_{\mathrm{BO}},
$$

where $T_{\mathrm{BO}}$ is expressed as follows:

$$
\begin{aligned}
T_{\mathrm{BO}} & =\left[1+\frac{1}{4}\left(\frac{1-b_{l}}{1-b_{l}^{(m+1)}}\right)\left(2 W_{0} \frac{1-\left(2 b_{1}\right)^{(m+1)}}{1-2 b_{l}}\right)\right. \\
& \left.-\left(\frac{3(m+1) b_{l}^{(m+1)}}{1-b_{l}}+\frac{3 b_{l}}{1-b_{l}}-\left(W_{0}+1\right)\right)\right],
\end{aligned}
$$

where $b_{l}=\max (\alpha,(1-\alpha) \beta)$.

The value of $\alpha$ and $\beta$ can be obtained from the following relations:

$$
\begin{aligned}
\alpha= & L\left(1-(1-\tilde{\tau})^{N-1}\right)(1-\alpha)(1-\beta)+L_{\mathrm{ack}} \\
& \cdot \frac{N \widetilde{\tau}(1-\widetilde{\tau})^{N-1}}{1-(1-\tilde{\tau})^{N}}\left(1-(1-\tilde{\tau})^{N-1}\right)(1-\alpha)(1-\beta), \\
\beta= & \frac{1-(1-\tilde{\tau})^{(N-1)}+N \widetilde{\tau}(1-\widetilde{\tau})^{N-1}}{2-(1-\tilde{\tau})^{N}+N \widetilde{\tau}(1-\tilde{\tau})^{N-1}},
\end{aligned}
$$

where $\tilde{\tau}$ is given by

$$
\widetilde{\tau}=(1+x)(1+\tilde{y}) \widetilde{b}_{0,0,0},
$$

where $\widetilde{b}_{0,0,0}, \tilde{y}$ and $\tilde{x}$ are given by

$$
\begin{aligned}
& \tilde{b}_{0,0,0}=\left(\frac{W_{0}}{2}(1+2 x)(1+\tilde{y})+L\left(1-x^{2}\right)(1+\tilde{y})\right. \\
& \left.\quad+L_{0} \frac{q_{0}}{1-q_{0}}\left(\tilde{y}^{2}\left(\tilde{y}^{n-1}+1\right)\right)+1\right)^{-1}, \\
& \tilde{y}=\left(1-\left(1-\tilde{\tau}^{N-1}\right)\right)\left(1-x^{2}\right), \\
& \tilde{x}=\alpha+(1-\alpha) \beta .
\end{aligned}
$$

The reliability $(R)$ of communication is defined as the probability that the $\mathrm{CH}$ receives the packet successfully. The formula for the reliability is

$$
R \approx 1-x^{(m+1)}(1+\tilde{y})-\widetilde{y}^{(n+1)} .
$$

Finally, we compute the total power consumption $(P)$ using the following formula

$$
\begin{aligned}
P \approx & \frac{P_{i} \tilde{\tau}}{2}\left(\frac{(1-x)\left(1-(2 x)^{(m+1)}\right)}{(1-2 x)\left(1-x^{(m+1)}\right)} W_{0}-1\right) \\
+ & P_{\mathrm{SC}}(2-\alpha) \tilde{\tau}+(1-\alpha)(1-\beta) \\
& \cdot \tilde{\tau}\left(P_{t} L+P_{i}+L_{\mathrm{ack}}\left(P_{r}\left(1-P_{C}\right)+P_{i} P_{C}\right)\right),
\end{aligned}
$$

where $P_{i}, P_{\mathrm{SC}}, P_{t}$, and $P_{r}$ are the average power consumption in idle-listen, channel sensing, transmit, and receiving states, 
TABLE 2: Optimization parameters.

\begin{tabular}{|c|c|c|}
\hline Parameter & Definition & Expression/value \\
\hline$N$ & $\begin{array}{l}\text { The number of nodes available in a single } \\
\text { cluster }\end{array}$ & $\begin{array}{l}\text { Assumed in the scenario under } \\
\text { study }\end{array}$ \\
\hline$L$ & The size of the frames sent & $\begin{array}{l}\text { Assumed in the scenario under } \\
\text { study }\end{array}$ \\
\hline$L_{\text {ack }}$ & The size of the ACK frame & $\begin{array}{l}\text { Assumed in the scenario under } \\
\text { study }\end{array}$ \\
\hline$S_{b}$ & $\begin{array}{l}\text { The time unit (aUnitBackoffPeriod) } \\
\text { defined in the 802.15.4 standard [1] }\end{array}$ & 20 symbols \\
\hline$W_{0}$ & Size of the backoff window & $\begin{array}{l}2^{\text {macMinBE }}, \text { macMinBE is between } \\
0 \text { and } \operatorname{mac} \operatorname{Max} B E(3-8)\end{array}$ \\
\hline$\alpha$ & $\begin{array}{l}\text { The probability of finding the wireless } \\
\text { medium busy during CCA1 }\end{array}$ & (r) \\
\hline$\beta$ & $\begin{array}{l}\text { The probability of finding the wireless } \\
\text { medium busy during CCA } 2\end{array}$ & $(4)$ \\
\hline$\tilde{\tau}$ & Approximated $\tau$ & (5) \\
\hline$\widetilde{b}_{0,0,0}$ & $\begin{array}{l}\text { The approximate probability of being in } \\
\text { the first backoff stage with both backoff } \\
\text { and retransmission counters in their } \\
\text { initial states }\end{array}$ & (6) \\
\hline$\tilde{y}$ & Approximated $y$ & $(7)$ \\
\hline$\tau$ & $\begin{array}{l}\text { The probability that a node starts CCA1 } \\
\text { in any time slot }\end{array}$ & $(1+x)(1+y) b_{0,0,0}$ \\
\hline$m$ & macMaxCSMABackoffs & $0-5$ (default: 4$)$ \\
\hline$x$ & $\begin{array}{l}\text { The probability of finding either CCA1 or } \\
\text { CCA } 2 \text { busy }\end{array}$ & (8) \\
\hline$y$ & $\begin{array}{l}\text { The probability of having a collision } \\
m+1 \text { times }\end{array}$ & $P_{C}\left(1-x^{m+1}\right)$ \\
\hline$P_{C}$ & The probability of collision & (11) \\
\hline$n$ & macMaxFrameRetries & 0-7 (Default 3) \\
\hline
\end{tabular}

respectively, and $P_{C}$ is the probability of collision and is given by

$$
P_{C}=1-(1-\widetilde{\tau})^{N-1}
$$

Based on (2), (9), and (10) our aim is to solve two important optimization problems for each member of a WSN cluster:

$$
\begin{array}{ll}
\underset{k}{\operatorname{minimize}} & T(k) \\
\text { subject to } & R_{i j} \geq \xi_{n}, \\
& n>0 \\
& \alpha, \beta, \tilde{\tau} \geq 0 \\
& P_{i}, P_{\mathrm{SC}}, P_{t}, P_{r}>0 \\
& P_{C} \geq 0 \\
& 0 \leq W_{0} \leq \operatorname{macMaxBE} \\
& 3 \leq \operatorname{macMaxBE} \leq 8 \\
& 0 \leq m \leq 5,
\end{array}
$$

$$
\begin{array}{cl}
\underset{k}{\operatorname{minimize}} & P(k) \\
\text { subject to } & R_{i j} \geq \xi_{n}, \\
& n>0 \\
& \alpha, \beta, \tilde{\tau} \geq 0 \\
& P_{i}, P_{\mathrm{SC}}, P_{t}, P_{r}>0 \\
& P_{\mathrm{C}} \geq 0 \\
& 0 \leq W_{0} \leq \operatorname{macMaxBE} \\
& 3 \leq \operatorname{macMaxBE} \leq 8 \\
& 0 \leq m \leq 5 .
\end{array}
$$

In Problem (12), the objective function minimizes the overall latency seen by every node $k$, in a cluster of $N$ nodes, given a minimum reliability $(\xi)$. In developing Problem (12), we assumed that an energy harvesting technique is used (see [10] for examples on such a technique). Thus, no constraints on power constraints are assumed. In Problem (13), however, the objective function minimizes the total power consumed by every node $k$, in a cluster of $N$ nodes, given the minimum reliability $\xi$. 
TABLE 3: Optimization parameters.

\begin{tabular}{lc}
\hline Parameter & Value \\
\hline$P_{i}$ & $0.8 \mathrm{~mW}$ \\
$P_{\text {SC }}$ & $40 \mathrm{~mW}$ \\
$P_{t}$ & $30 \mathrm{~mW}$ \\
$P_{r}$ & $40 \mathrm{~mW}$ \\
macMaxFrameRetries & 2 to 7 \\
macMaxCSMABackoffs & 2 to 5 \\
macMinBE & 3 to 8 \\
$L$ & 120 bytes \\
$L_{\text {ack }}$ & 12 byte \\
\hline
\end{tabular}

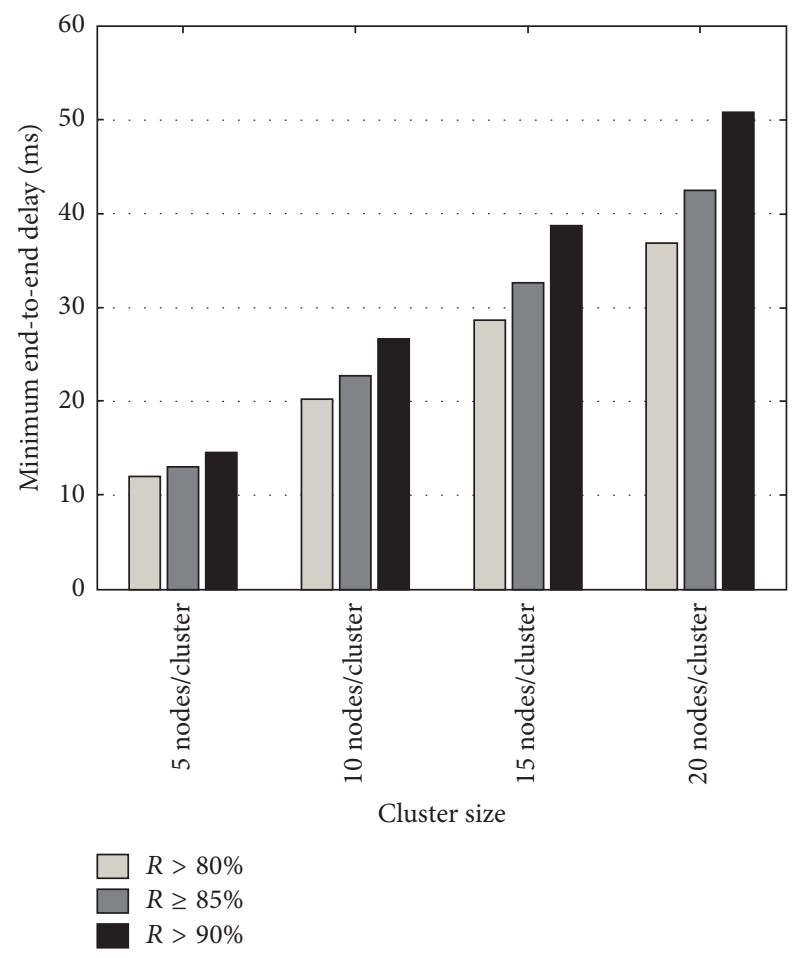

Figure 1: The minimum latency as a function of the number of nodes in a cluster.

\section{Results and Discussions}

We use LINGO 15.0 software [21] to solve Problems (12) and (13) that were developed in Section 4. The parameters that we used in solving both of the optimization problems are listed in Table 3. Figure 1 illustrates the results of solving Problem (12) with $\xi$ set to $80 \%, 85 \%$, and $90 \%$, respectively. At $\xi=80 \%$, we can see that a cluster of 20 nodes guarantees a minimum endto-end latency of almost $36.81 \mathrm{~ms}$. At $\xi=85 \%$, for the same cluster size, the minimum latency becomes almost 42.37 . Finally, the minimum latency becomes $50.66 \mathrm{~ms}$ at $\xi=90 \%$. These results can benefit several smart grid applications that can tolerate up to $250 \mathrm{~ms}$ of latency (See Section 1). Based on Table 4, we can see that Problem (12) does have feasible solutions if the cluster size is increased to 30 nodes. With
TABle 4: Minimum delays with $(\mathscr{I} \mathcal{S})$.

\begin{tabular}{lccc}
\hline Cluster size & $R>80 \%$ & $R \geq 85 \%$ & $R>90 \%$ \\
\hline 25 & 45.08 & 52.15597 & $\mathscr{I} \mathcal{S}$ \\
30 & 53.34531 & 61.94045 & $\mathscr{I} \mathcal{S}$ \\
35 & 61.6105 & $\mathscr{I} \mathcal{S}$ & $\mathscr{I} \mathcal{S}$ \\
\hline
\end{tabular}

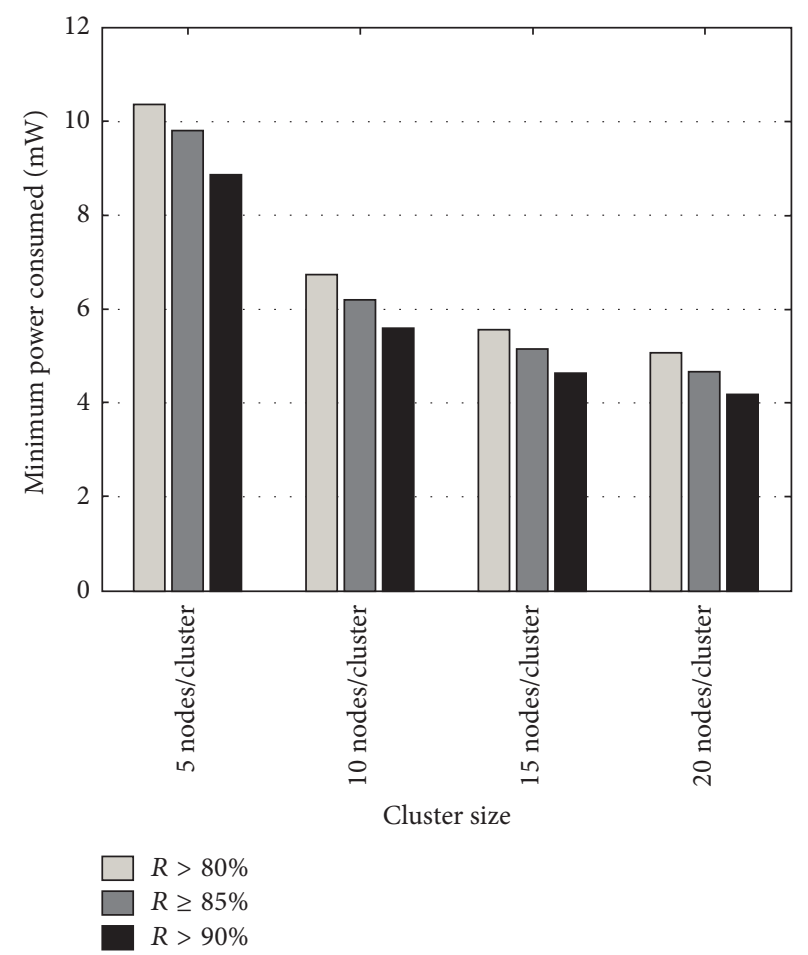

FIGURE 2: The minimum power consumption as a function of the number of nodes in a cluster.

the latter size, the minimum latency is $53.34 \mathrm{~ms}$ at $\xi=80 \%$ and $61.94 \mathrm{~ms}$ at $\xi=85 \%$. Evidently, we can still support the aforementioned smart grid applications by increasing the size of the cluster to 30 nodes. Table 4 also shows that we will hit infeasible solutions $(\mathscr{I} \mathcal{S})$ for Problem (12) in case we set $\xi=$ $90 \%(N=35)$ or we set $\xi=90 \%(N=25,30$, or 35$)$. All these findings that relate the size of the cluster, the minimum reliability, and the expected minimum latency can help WSN designers to properly cluster the network.

The results of solving Problem (13) are depicted in Figure 2. Again, the results are generated under $\xi$ being $80 \%$, $85 \%$, or $90 \%$. The figure clearly shows that as $N$ increases the consumption of power at any node decreases. This can be explained by noticing that as the number of nodes in a cluster increases, the nodes will suffer from more packet collisions (11). As a result, nodes will back off, after repeated collisions, for extended periods of time. This means that transmission and reception activities (which are the main contributors to power consumption) will be lower, and that leads to lower overall power consumption in the cluster. To support this observation, we can see that as $N$ increased LINGO started to pick the value 8 for the macMinBE parameter, which is the maximum allowed value for macMinBE (see Table 2). 
TABLE 5: Minimum power consumption with $(\mathscr{I} \mathcal{S})$.

\begin{tabular}{lccc}
\hline Cluster size & $R>80 \%$ & $R \geq 85 \%$ & $R>90 \%$ \\
\hline 25 & 4.741881 & 4.379537 & $\mathscr{I} \mathcal{S}$ \\
30 & 4.53222 & 4.186752 & $\mathscr{I} \mathcal{S}$ \\
35 & 4.384003 & $\mathscr{I} \mathcal{S}$ & $\mathscr{I} \mathcal{S}$ \\
\hline
\end{tabular}

In other words, as the size of the cluster increased, the optimization technique used by LINGO kicks in to try to minimize the objective function. It is noteworthy that at $\xi=$ $90 \%$ and $N=20$ the power consumption is so low $(4.2 \mathrm{~mW})$. As in Table 4, Table 5 highlights the situations where we can hit infeasible solutions for Problem (13).

\section{Conclusions}

In this paper we tackled two important optimization problems in clustered WSNs. In the first problem we worked on minimizing the end-to-end latency under constraints on communication reliability. Under the same constraints, the second problem aimed at minimizing the total power consumption. Important applications, like latency-critical monitoring applications in the smart grid, can benefit from such a study. By solving these optimization problems we found that a WSN with clusters of sizes up to 30 nodes can achieve the latency requirements of important smart grid applications. We also found that a WSN of clusters of 20 nodes can operate at low power consumption while obeying a minimum reliability of $90 \%$. With the widespread usage of smart grids deployments (at the global scale) to improve power distribution to consumers, our findings in this paper support the case of deploying WSNs in the context of the smart grid. The WSNs can bring the smart grid a richness of features while obeying the latter's constraints and requirements.

\section{Conflicts of Interest}

The authors declare that there are no conflicts of interest regarding the publication of this paper.

\section{References}

[1] IEEE std 802.15.4-2006 (revision of IEEE std 802.15.4-2003), https://standards.ieee.org/findstds/standard/802.15.4-2006 .html.

[2] I. F. Akyildiz, T. Melodia, and K. R. Chowdury, "Wireless multimedia sensor networks: a survey," IEEE Wireless Communications, vol. 14, no. 6, pp. 32-39, 2007.

[3] M. Khanafer, M. Guennoun, and H. T. Mouftah, "A survey of beacon-enabled IEEE 802.15.4 MAC protocols in wireless sensor networks," IEEE Communications Surveys \& Tutorials, vol. 16, no. 2, pp. 856-876, 2014.

[4] L. M. Borges, F. J. Velez, and A. S. Lebres, "Survey on the characterization and classification of wireless sensor network applications," IEEE Communications Surveys and Tutorials, vol. 16, no. 4, pp. 1860-1890, 2014.
[5] I. Al-Anbagi, M. Erol-Kantarci, and H. T. Mouftah, "A survey on cross-layer quality-of-service approaches in WSNs for delay and reliability-aware applications," IEEE Communications Surveys and Tutorials, vol. 18, no. 1, pp. 525-552, 2016.

[6] P. Park, P. Di Marco, C. Fischione, and K. H. Johansson, "Modeling and optimization of the IEEE 802.15.4 protocol for reliable and timely communications," IEEE Transactions on Parallel and Distributed Systems, vol. 24, no. 3, pp. 550-564, 2013.

[7] P. Park, S. C. Ergen, C. Fischione, and A. Sangiovanni-Vincentelli, "Duty-cycle optimization for IEEE 802.15.4 wireless sensor networks," ACM Transactions on Sensor Networks, vol. 10, no. 1, article 12, 2013.

[8] J. H. Ortiz, "Telecommunications Networks - Current Status and Future Trends," InTech, 2012.

[9] D. Incebacak, R. Zilan, B. Tavli, J. M. Barcelo-Ordinas, and J. Garcia-Vidal, "Optimal data compression for lifetime maximization in wireless sensor networks operating in stealth mode," Ad Hoc Networks, vol. 24, pp. 134-147, 2015.

[10] F. K. Shaikh and S. Zeadally, "Energy harvesting in wireless sensor networks: a comprehensive review," Renewable and Sustainable Energy Reviews, vol. 55, pp. 1041-1054, 2016.

[11] K. C. Budka, J. G. Deshpande, M. Thottan et al., "Communication networks for smart grids," in Computer Communications and Networks, Computer Communications and Networks, Springer, 2014.

[12] S. Mohrehkesh and M. C. Weigle, "Optimizing communication energy consumption in perpetual wireless nanosensor networks," in Proceedings of the 2013 IEEE Global Communications Conference, GLOBECOM 2013, pp. 545-550, USA, December 2013.

[13] H. M. Ammari, "On the energy-delay trade-off in geographic forwarding in always-on wireless sensor networks: a multiobjective optimization problem," Computer Networks, vol. 57, no. 9, pp. 1913-1935, 2013.

[14] K. Suto, H. Nishiyama, N. Kato, and CW. Huang, "An energyefficient and delay-aware wireless computing system for industrial wireless sensor networks," IEEE Access, vol. 3, pp. 10261035, 2015

[15] D. He, G. Mujica, J. Portilla, and T. Riesgo, "Modelling and planning reliable wireless sensor networks based on multi-objective optimization genetic algorithm with changeable length," Journal of Heuristics, vol. 21, no. 2, pp. 257-300, 2015.

[16] A. E. Zonouz, L. Xing, V. M. Vokkarane, and Y. L. Sun, "Reliability based optimization in hybrid wireless sensor networks," in Proceedings of the 61st Annual Reliability and Maintainability Symposium, RAMS 2015, pp. 1-7, USA, January 2015.

[17] M. Dong, K. Ota, A. Liu, and M. Guo, "Joint optimization of lifetime and transport delay under reliability constraint wireless sensor networks," IEEE Transactions on Parallel and Distributed Systems, vol. 27, no. 1, pp. 225-236, 2016.

[18] I. Al-Anbagi, M. Erol-Kantarci, and H. T. Mouftah, "Priorityand delay-aware medium access for wireless sensor networks in the smart grid," IEEE Systems Journal, vol. 8, no. 2, pp. 608-618, 2013.

[19] G. Bianchi, "Performance analysis of the IEEE 802.11 distributed coordination function," IEEE Journal on Selected Areas in Communications, vol. 18, no. 3, pp. 535-547, 2000.

[20] P. Park, P. Di Marco, P. Soldati, C. Fischione, and K. H. Johansson, "A generalized Markov chain model for effective analysis of slotted IEEE 802.15.4," in Proceedings of the IEEE 6th 
International Conference on Mobile Adhoc and Sensor Systems (MASS '09), pp. 130-139, Macau, China, October 2009.

[21] E. Goodarzi, M. Ziaei, and E. Z. Hosseinipour, "Optimization analysis using LINGO and MATLAB," in Introduction to Optimization Analysis in Hydrosystem Engineering, vol. 25, pp. 149193, Springer, 2014. 


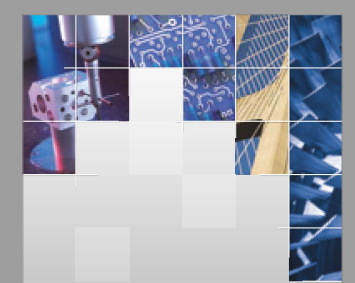

\section{Enfincering}
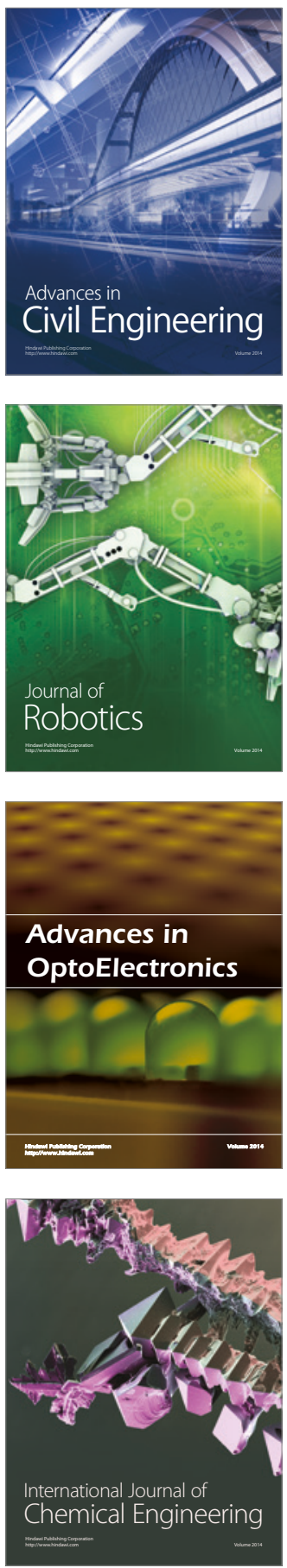

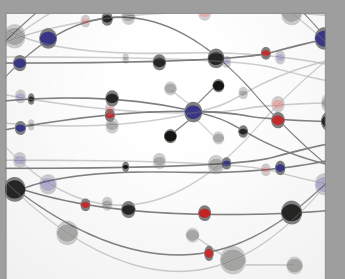

The Scientific World Journal

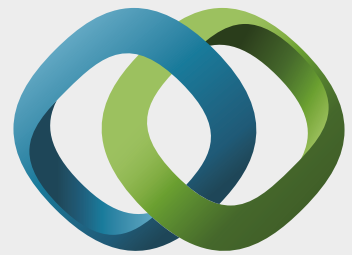

\section{Hindawi}

Submit your manuscripts at

https://www.hindawi.com
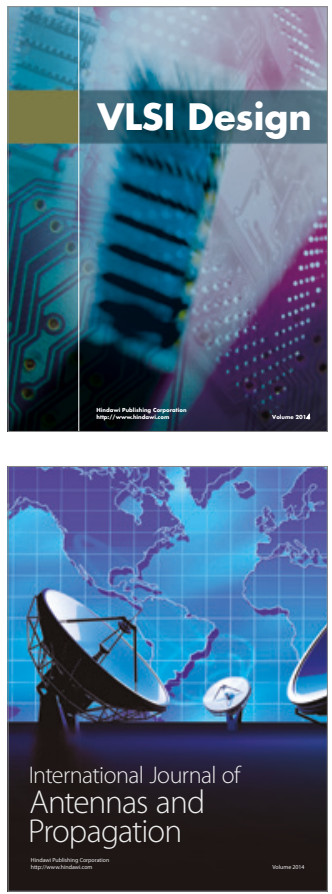

\section{Rotating}

Machinery
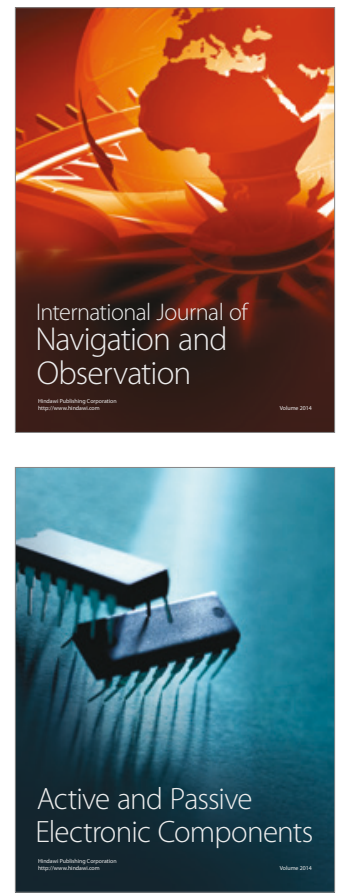
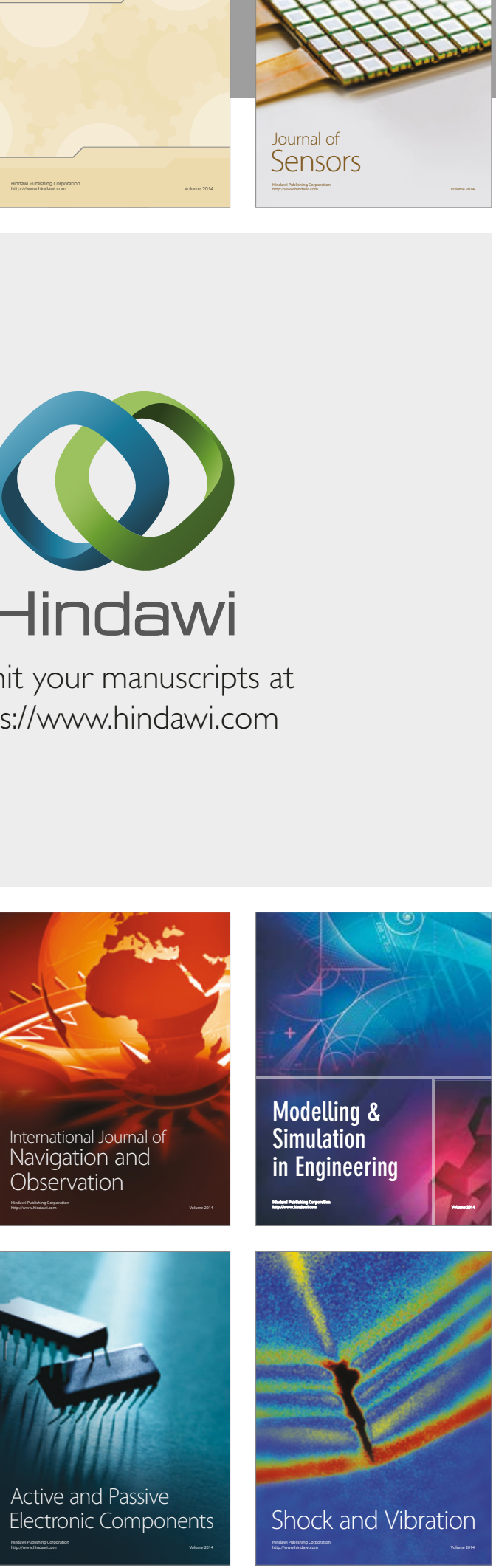
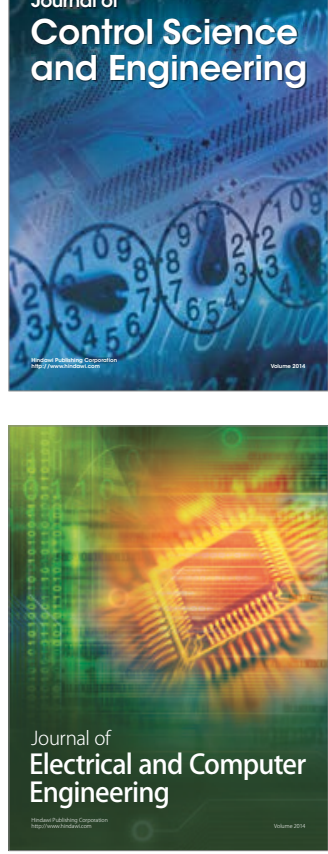

Distributed

Journal of

Control Science

and Engineering
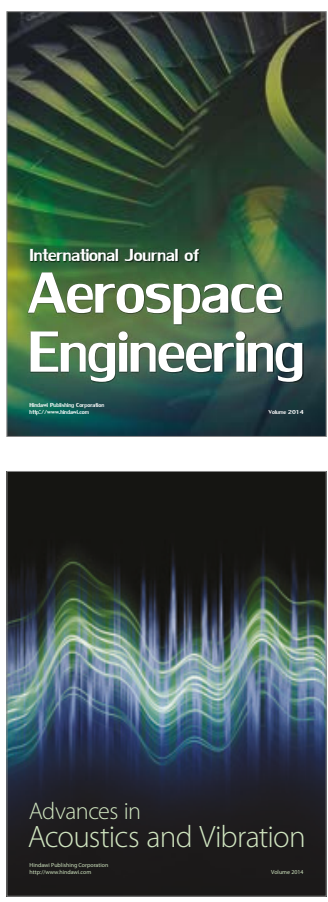

Sensor Networks 